\title{
A phase-field simulation study of the migration of recrystallization boundaries
}

\author{
Nele Moelans* \\ Dept. Metallurgy and Materials Engineering, KU Leuven, \\ Kasteelpark Arenberg 44, box 2450, B-3001 Leuven, Belgium \\ Andy Godfrey ${ }^{\dagger}$ \\ Key Laboratory of Advanced Materials (MOE), \\ School of Materials Science and Engineering, \\ Tsinghua University, Beijing 100084, People's Republic of China \\ Yubin Zhang \} ^ { \ddagger } \text { and Dorte Juul Jensen } { } ^ { \S } \\ Danish Chinese Center for Nanometals, \\ Section for Materials Science and Advanced Characterization, \\ Department of Wind Energy, Risø Campus, \\ Technical University of Denmark, DK-4000 Roskilde, Denmark
} (Dated: July 19, 2013) 


\begin{abstract}
We present simulation results based on a phase-field model that describes the local migration of recrystallization boundaries into varying deformation energy fields. An important finding from the simulations is that the overall migration rate of the recrystallization front can be considerably affected by the variations in the deformed microstructure, resulting in 2 regimes. For variations with low amplitude, the overall boundary velocity scales with the average stored deformation energy density. This behavior is in agreement with generally accepted theories of recrystallization. For larger amplitudes, however, the velocity scales with the maximum of the deformation energy density along the variation, resulting in a considerably larger velocity than that obtained from standard recrystallization models. The shape of the migrating grain boundary highly depends on the local characteristics of the varying stored deformation energy field. For different deformation energy fields, the simulation results are in good qualitative agreement with experiments and add information which cannot be directly derived from experiments.
\end{abstract}

PACS numbers: 5

Keywords: recrystallization, microstructure, Phase-Field method 


\section{INTRODUCTION}

Deformation of a metal is often followed by an annealing step, leading to recrystallization by which the deformed material is replaced by new almost defect free grains that grow at the expense of the deformed matrix. The driving force for recrystallisation arises from the excess dislocation density and other defects that were generated by the deformation process and stored in the deformed metal. Generally, it is assumed that, in the absence of obstacles such as pinning precipitates, recrystallization boundaries move smoothly with a velocity $v_{g b}$ proportional to the driving force for recrystallization $P_{\text {rex }}$ :

$$
v_{g b}=M P_{r e x},
$$

where $M$ is the grain boundary mobility.

A new trend in recrystallization studies is to consider local phenomena instead of using traditional approaches that consider on the average behaviour. A consideration of the effect of local variations in the deformation microstructures is therefore essential. Extensive studies ${ }^{1}$ have shown that such local variations exist on all scales from the macroscopic sample scale, where inhomogeneous deformation or forming may lead to substantially different microstructures in different parts of the sample, to the local grain scale. In the latter case, grains of different orientations are known to deform by different slip systems leading to different deformation microstructures ${ }^{2}$, and even within grains, the organization of dislocation boundaries can show significant local variations ${ }^{3-5}$. The driving force for recrystallization will accordingly vary along the recrystallization boundary. Recent experiments have also shown that grain boundary movement during recrystallization of samples deformed to both low and high strains is often very irregular, even in pure materials ${ }^{6-10}$. It is observed that individual grain boundary segments move with different rates and often show a stop-and-go type of movement. In-situ observations and ex-situ microstructural studies of recrystallization in materials with medium to high stacking fault energy, such as $\mathrm{Ni}$ and $\mathrm{Al}$, clearly show the presence of protrusions (these are parts of the boundary that have advanced more than other parts) and retrusions (parts of the boundary that are behind) of a few up to tens of $\mu \mathrm{m}$ along 
recrystallization boundaries (see for example the picture shown in Fig. 1). Moreover the boundary profile is dynamic, such that protrusions can disappear or transform into retrusions and vice-versa as a migrating recrystallization boundary moves into a deformed matrix.

Zhang et al. ${ }^{11,12}$ have measured the curvature of protrusions and retrusions along recrystallization boundaries and related the values to the local stored deformation energy in front of the moving boundary. Although, on average, large variations in stored deformation energy seem to result in protrusions and retrusions with high curvatures, the size and shape of the protrusions/retrusions seems to be correlated in a more complex way to the spatial variations in the stored deformation energy ${ }^{11}$. Another important conclusion from these studies was that the magnitude of the local curvature based driving force at protrusions and retrusions is comparable to that of the stored energy within the deformed microstructure. The presence of protrusions/retursion thus can be considered to provide locally an extra driving (or dragging) force which should be accounted for when calculating the local migration rate of the recrystallization boundary:

$$
v_{g b}=M\left(P_{\text {rex }}+P_{\text {curve }}\right)
$$

where $P_{\text {rex }}$ is the local driving force from the stored energy and $P_{\text {curve }}$ is the driving force due to the local curvature of the boundary.

Several simulation methodologies have been applied to study the effect of local variations in the stored deformation energy on the possibility for the formation of protrusions during recrystallization. Martorano et al. studied the formation of protrusions ${ }^{10,13}$ by numerical integration of equation (2) considering the movement of a boundary into a deformation field with a 1-d sinusoidally varying energy field (to represent the strain field of the dislocation walls). It was found that for the considered variations, a steady-state regime could be reached, for which the grain boundary shape was characterized in detail. Furthermore, molecular dynamics simulations have shown that the interactions of a recrystallization boundary with individual dislocations and local subboundaries can result in irregular grain boundary motion and the formation of local protrusions/retrusions over a few atomic distances ${ }^{14,15}$. Irregular boundary migration was also obtained using a phase-field model in which long-range strain fields were used to account for the stored elastic energy associated with dislocations in a deformed material ${ }^{16}$. 
None of these approaches however can be easily extended to reproduce the full complexity of the grain boundary migration observed in recrystallization experiments. Calculation of the local grain boundary position as a function of time by integration of equation (2) for a deformation energy field $P_{r e x}$ with local variations of an arbitrary form appears to be an extremely complex moving boundary problem ${ }^{13}$, and no analytical solutions of equation (2) are available, even for the case of a simple sinusoidally varying field. It is also not clear to what extent the extra curvatures associated with the presence of protruded and retruded parts along the recrystallization boundary affect the overall recrystallization kinetics on larger length and time scales ${ }^{12}$.

As an alternative approach, we present a phase-field model to simulate the formation of protrusions/retrusions during migration of a recrystallization boundary into a deformed microstructure containing variations in stored energy that do not vary with time. The deformation energy field for the model is constructed to represent the characteristics of typical microstuctures deformed to mediumto-large strains. The local driving force for migration of the recrystallization front is treated in a similar way as in the model of Martorano et al. However, an important advantage of using a phase-field description is that the model can treat easily more realistic situations, such as different forms of variations in the deformation energy field, 3-d microstructures ${ }^{17}$ and the effect for example of anisotropic grain boundary properties or a structure dependent grain boundary mobility.

Our long-term aim is to obtain a systematic understanding of the effect of local variations of various forms in the stored deformation energy field on the formation of protrusions and retrusions with certain characteristics along the recrystallization front and to ascertain their effect on the overall migration rate of the recrystallization front. Such insights are difficult to obtain experimentally, although they are needed for guiding and interpreting further experimental studies. In the current paper, we mainly consider simulations using deformation fields with one-dimensional variations. It will be shown that these variations have strong effects on the overall boundary migration rate, on the formation of local protrusions and retrusions on the steady state moving boundary and that asymmetric shapes of the protrusion-retrusion profile may evolve. 


\section{MODEL DESCRIPTION}

\section{A. Phase field model}

The phase-field model in this study is based on the grain growth model of Chen and Yang $^{18}$, using the modified free energy formulation and parameter relations of Moelans et al. ${ }^{19,20}$. An additional term is introduced to represent the spatial variation of the energy in the deformed microstructure. For the results presented in this paper, a 2-dimensional system is considered comprising one recrystallizing grain growing into the deformed matrix of a single grain. Two order parameters $\eta_{\text {rex }}(\mathrm{x}, \mathrm{y}, t)$ and $\eta_{\text {def }}(\mathrm{x}, \mathrm{y}, t)$, which are continuous functions of the spatial coordinates $\mathrm{x}$ and $\mathrm{y}$ and time $t$, are used to distinguish the deformed and recrystallized parts of the system, with $\eta_{\text {rex }}=1$ and $\eta_{\text {def }}=0$ in the recrystallizing grain and $\eta_{\text {rex }}=0$ and $\eta_{\text {def }}=1$ in the deformed matrix. If only 2 domains (deformed and recrystallized) are considered in the simulations, a single-phase-field model using only 1 order parameter to distinguish between the recrystallized and deformed domain is sufficient. However, as we aim to extend our approach to polycrystalline structures in the future the model is constructed by representing the 2 types of grains (deformed and recrystallized grains) using 2 sets of order parameters, namely $\eta_{r e x, i}$ and $\eta_{d e f, k}$. It has been shown previously ${ }^{20}$ that the choice of model parameters for a 2-grain structure with a single grain boundary applies to the individual grain boundaries in polycrystalline structures represented by a large set of order parameters.

The thermodynamic free energy of the system $F$ is assumed to consist of contributions from grain boundary energy and from the stored deformation energy, namely $F=F_{g b}+F_{d e f}$. The formulation of the grain boundary energy contributions $F_{g b}$ is taken as

$$
\begin{aligned}
F_{g b}=\int_{\Omega}[ & \frac{6 \sigma_{g b}}{\ell_{g b}}\left(\frac{\eta_{\text {rex }}^{4}}{4}+\frac{\eta_{\text {def }}^{4}}{4}-\frac{\eta_{\text {rex }}^{2}}{2}-\frac{\eta_{\text {def }}^{2}}{2}+1.5 \eta_{\text {rex }}^{2} \eta_{\text {def }}^{2}+0.25\right) \\
& \left.+\frac{3}{8} \sigma_{g b} \ell_{g b}\left(\left(\nabla \eta_{\text {rex }}\right)^{2}+\left(\nabla \eta_{\text {def }}\right)^{2}\right)\right] \mathrm{d} \Omega
\end{aligned}
$$

where the integral is taken over the system domain $\Omega$. The first term is a polynomial which is minimal and equal to 0 for $\left(\eta_{\text {def }}, \eta_{\text {rex }}\right)=( \pm 1,0)$ or $(0, \pm 1)$, corresponding to the deformed and recrystallizing grains respectively. $\sigma_{g b}$ is grain boundary energy which can be taken from experimental measurements. $\ell_{g b}$ is the width of the diffuse interface zone associated 
with the boundary in the phase-field simulations ${ }^{19,20}$, which is chosen based on numerical considerations see section III.

The contribution from the stored deformation energy $F_{d e f}$ is introduced as

$$
F_{d e f}=\int_{\Omega} f_{d e f}(x, y) \frac{\eta_{d e f}^{2}}{\eta_{r e x}^{2}+\eta_{d e f}^{2}} \mathrm{~d} \Omega
$$

The factor $\eta_{\text {def }}^{2} /\left(\eta_{\text {rex }}^{2}+\eta_{\text {def }}^{2}\right)=\phi_{\text {def }}$ represents the local fraction of deformed material ${ }^{21}$, which equals 1 within the deformed grain and 0 in the recrystallizing grain. The diffuse interface zone is thus treated as a mixture of deformed and recrystallized material with a smoothly varying local fraction of each material. The local deformation energy density $f_{\text {def }}$ is a function of the spatial coordinates and can be of any continuous or discontinuous form that describes the deformation field well (see section II B).

The evolution equations of the order parameters are derived from the free energy functional $F=F_{g b}+F_{\text {def }}$ following the principles of linear non-equilibrium thermodynamics, namely

$$
\frac{\partial \eta_{\text {rex }}}{\partial t}=-\left(\frac{4}{3}\right)\left(\frac{M}{\ell_{g b}}\right) \frac{\delta F}{\delta \eta_{\text {rex }}} \quad \text { and } \quad \frac{\partial \eta_{\text {def }}}{\partial t}=-\left(\frac{4}{3}\right)\left(\frac{M}{\ell_{g b}}\right) \frac{\delta F}{\delta \eta_{d e f}}
$$

giving

$$
\begin{aligned}
\frac{\partial \eta_{\text {rex }}}{\partial t}=- & \left(\frac{4}{3}\right)\left(\frac{M}{\ell_{g b}}\right)\left[\frac{6 \sigma_{g b}}{\ell_{g b}}\left(\eta_{\text {rex }}^{3}-\eta_{\text {rex }}+3 \eta_{\text {rex }} \eta_{\text {def }}^{2}\right)\right. \\
& \left.-\frac{3}{4} \sigma_{g b} \ell_{g b} \nabla^{2} \eta_{\text {rex }}-\frac{2 \eta_{\text {rex }} \eta_{\text {def }}^{2}}{\left(\eta_{\text {rex }}^{2}+\eta_{\text {def }}^{2}\right)^{2}} f_{\text {def }}(\mathrm{x}, \mathrm{y})\right] \\
\frac{\partial \eta_{\text {def }}}{\partial t}=- & \left(\frac{4}{3}\right)\left(\frac{M}{\ell_{g b}}\right)\left[\frac{6 \sigma_{g b}}{\ell_{g b}}\left(\eta_{\text {def }}^{3}-\eta_{\text {def }}+3 \eta_{\text {def }} \eta_{\text {rex }}^{2}\right)\right. \\
& \left.-\frac{3}{4} \sigma_{g b} \ell_{g b} \nabla^{2} \eta_{\text {def }}+\frac{2 \eta_{\text {def }} \eta_{\text {rex }}^{2}}{\left(\eta_{\text {def }}^{2}+\eta_{\text {rex }}^{2}\right)^{2}} f_{\text {def }}(\mathrm{x}, \mathrm{y})\right],
\end{aligned}
$$

with $M$ the grain boundary mobility ${ }^{20}$. They therefore describe the evolution path with maximum energy dissipation.

In the results presented here the grain boundary energy and mobility are selected to be isotropic, in order to investigate directly the effect of the heterogeneous deformation field on the recrystallizing boundary. The model can, however, be extended to include anisotropy in grain boundary properties ${ }^{19,20}$. 


\section{B. Representation of the stored energy of deformation}

In many metals plastic deformation leads to a process of grain subdivision by the formation of dislocation boundaries with a range of boundary misorientation angles. These dislocation boundaries are most commonly arranged in the form of a cell block microstructure, where dislocation boundaries of larger misorientation angles delineate rectangular volumes in which smaller interconnecting dislocation boundaries are formed ${ }^{3,5,22}$. In the ideal case such boundaries have an energy related to the boundary misorientation angle by the Read-Shockley equation $^{23}$, and are free from long-range stress fields. In practice, however, small long range stresses, of up to the level of about half of the flow stress may be present, decaying approximately as $\ln (1 / \mathrm{r})$ with distance (r) from each boundary. Additionally some loose dislocations (not incorporated into dislocation boundaries) are generally present in such deformed microstructures. The deformation microstructure also exhibits a strong orientation dependence such that within a single original grain cell block boundaries are approximately parallel to one another. Local variations in the spacing between and misorientation angle across the dislocation boundaries are also typically observed. These features are illustrated in Fig. 2.

As the simplest model to capture the variation in stored energy of such deformation microstructure we consider the energy field to take the form of a sinusoidal function with maxima at the positions of the cell block boundaries and a minimum energy in the cell block interior. In this case the energy field is described by

$$
f_{\text {def }}=A_{d e f}\left(1+\sin \left(\frac{2 \pi}{\lambda_{d e f}} \mathrm{x}\right)\right)+C_{d e f},
$$

with amplitude $A_{d e f}$, wavelength $\lambda_{d e f}$ and a minimum energy (offset) $C_{d e f}$. This energy field is referred to hereafter as type 's'. As a more realistic model of the variation deformation energy we also consider two asymmetric functions where the maximum stored energy is assumed to be more concentrated in regions near some cell block boundaries. These energy fields are described by:

$$
f_{\text {def }}=A_{\text {def }}\left(1+\sin \left(\frac{3 \pi}{\lambda_{\text {def }}} \mathrm{x}\right)\right)+C_{\text {def }} \quad \text { for } \quad 0<\mathrm{x} \leq \lambda_{\text {def }} / 3
$$




$$
=A_{d e f}\left(1+\sin \left(\frac{1.5 \pi}{\lambda_{d e f}}\left(\mathrm{x}+\frac{\lambda_{d e f}}{3}\right)\right)\right)+C_{\text {def }} \quad \text { for } \quad \lambda_{\text {def }} / 3<\mathrm{x} \leq \lambda_{\text {def }}
$$

(referred to as 'a1') and

$$
\begin{aligned}
f_{d e f} & =A_{d e f}\left(1+\sin \left(\frac{3 \pi}{\lambda_{d e f}} \mathrm{x}\right)\right)+C_{\text {def }} \quad \text { for } \quad 0<\mathrm{x} \leq \lambda_{\text {def }} / 3 \\
& =A_{\text {def }}\left(1+0.5 \sin \left(\frac{1.5 \pi}{\lambda_{\text {def }}}\left(\mathrm{x}+\frac{\lambda_{\text {def }}}{3}\right)\right)\right)+C_{\text {def }} \quad \text { for } \quad \lambda_{\text {def }} / 3 \leq \mathrm{x}<\lambda_{\text {def }}
\end{aligned}
$$

(referred to as 'a2'). All three are sketched in Fig. 3. For variations 's' and 'a2', the average stored deformation energy equals $A_{d e f}+C_{d e f}$. For variation 'a1', the average stored deformation energy is given by $A_{d e f}(1-2 /(3 \pi))+C_{\text {def }}$.

These descriptions are still idealized in that they do not include the full extent of variations in the magnitude of the boundaries misorientation angle across each cell block boundary (and hence the stored energy) or in the spacing between cell block boundaries. They nevertheless capture the basic characteristics of expected spatial variations in stored energy in a deformation microstructure in a way that allows examination of the key parameters of $A_{d e f}$, wavelength $\lambda_{d e f}$, and a minimum energy (offset) $C_{d e f}$ on the local migration of a recrystallizing boundary.

\section{SIMULATION INPUT, NUMERICAL DETAILS AND VALIDATION}

The migration of an initially planar grain boundary parallel to the x-axis into the varying deformation fields shown in Fig. 3 will be studied in the simulations.

Based on experimental information ${ }^{5,11,12,24}$ for aluminium and nickel, the amplitude of the variations $A_{\text {def }}$ was varied between 0.1 and $20 \mathrm{MJ} / \mathrm{m}^{3}$ and the wavelength $\lambda_{\text {def }}$ was taken 1.6, 3.2 and $6.4 \mu \mathrm{m}$ for the symmetrical variation 's' and 2.4 and $4.8 \mu \mathrm{m}$ for the asymmetrical variations 'a1' and 'a2', using grain boundary energies of $\sigma_{g b}=0.32 \mathrm{~J} / \mathrm{m}^{2}$ (aluminium) and $\sigma_{g b}=0.87 \mathrm{~J} / \mathrm{m}^{2}$ (nickel). The value of the offset $C_{d e f}$ was arbitrarily taken equal to 0 , 0.34 and $2.01 \mathrm{MJ} / \mathrm{m}^{3}$ merely to verify its effect. The grain boundary mobility only affects the time-scaling of the simulations. Therefore rather than using experimental values for $M$, which are strongly dependent on solute content, we used a value of $M=1 \mathrm{~m}^{4} /(\mathrm{MJs})$.

Based on numerical considerations, the remaining model and simulation parameters were taken so that $6 \sigma_{g b}=\ell_{g b}, \ell_{g b}=\sqrt{9.6} \Delta x,\left|f_{d e f, \max }\right| \ell_{g b} \leq 0.6 \sigma_{g b}$ and $M \Delta t=0.0375 \ell_{g b}$ 
where $\Delta x$ and $\Delta t$ are the discrete grid spacing and time step respectively. It has been shown previously that for these values grain boundary motion is resolved with a relative (numerical) error smaller than $5 \%^{20}$. This was determined by comparison of the shrinkage rate of a circular grain under purely curvature driven boundary migration with the value expected from analytical theory. A similar accuracy was found for the case of migration of a flat boundary under a homogeneous driving force ${ }^{25}$.

To assess for the presence of artificial effects introduced by the use of a particular numerical scheme, two discretization schemes were applied to solve equations (6) and (7), namely a Fourier-based spectral method with semi-implicit time stepping as described in ${ }^{26,27}$ and a standard finite difference discretisation with a central 5-point scheme for the spatial Laplacian and explicit time stepping. With the Fourier spectral method, periodic boundary conditions are required along both, the $\mathrm{x}-$ and $\mathrm{y}^{-}$, directions. The simulation domain accordingly contains 2 boundaries which however behave identically. With the finite-difference scheme, periodic boundary conditions were applied along the x-direction and Neumann (zero-gradient) conditions along the y-direction. The movement of only 1 boundary was thus considered in these simulations. Both techniques gave very similar simulation results for the considered model parameter values, grid spacings and time stepping. Moreover, comparison of the steady-state shape and migration rate of a boundary in phase-field simulations with a symmetrical 1-dimensional sinusoidally varying deformation energy field with that obtained using the sharp interface analysis of Martorano et al. ${ }^{13}$ (solved using an extremely fine discretization ) shows that the phase-field simulation results have indeed a numerical error smaller than $5 \%$ for the chosen model parameter values.

For all simulations, the local positions $d_{i}$ (in the y-direction) of the grain boundary at all discrete grid points $i$ along the x-direction were extracted at different time steps as

$$
d_{i}=\Delta x \sum_{j}\left[1-\phi_{\text {def }}\left(x_{i}, y_{j}\right)\right]
$$

with $\phi_{\text {def }}=\eta_{\text {def }}^{2} /\left(\eta_{\text {rex }}^{2}+\eta_{\text {def }}^{2}\right)$ the local fraction of deformed material as defined before (section II A) evaluated at all grid points $\left(x_{i}, y_{j}\right)$ and with $\sum_{j}$ a sum taken over all discrete grid points along the $\mathrm{y}$-direction. The local curvatures were calculated from the local positions $d_{i}$ as

$$
\frac{1}{\rho_{i}}=\frac{\left(d^{\prime \prime}\right)_{i}}{\left[1+\left(d^{\prime}\right)_{i}^{2}\right]^{1.5}},
$$

with $\left(d^{\prime}\right)_{i}=\left[d_{i+1}-d_{i}\right] / \Delta x$ and $\left(d^{\prime \prime}\right)_{i}=\left[\left(d^{\prime}\right)_{i}-\left(d^{\prime}\right)_{i-1}\right] / \Delta x$. 
To validate the curvature calculations and study the effect of the presence of retrusions on the shape of the protrusions and the grain boundary migration velocity, a limited number of simulations were performed assuming a stored deformation energy field of the form shown in Fig. 3a, but considering only the protrusion region, thus considering a system with width $\lambda_{\text {def }} / 2$. The finite difference discretization was here applied with different types of boundary conditions along the boundaries $\mathrm{x}=0$ and $\mathrm{x}=\lambda_{\text {def }} / 2$ and zero-gradient conditions along the $y$-direction.

In one type of test simulations, referred to as 'static', the ends of the boundary are pinned by fixing the values of the order-parameters at $\mathrm{x}=0$ and $\mathrm{x}=\lambda_{\text {def }} / 2$. For this configuration, the grain boundary obtains an equilibrium shape after a short simulation time, and obeys the force balance equation along the y-direction $f_{d e f}(\mathrm{x})=\sigma_{g b} /|\rho(\mathrm{x})|$, as expected. Simulated curvatures are compared with the expected curvatures along the boundary in Fig. 4. The deviation is always much smaller than $5 \%$.

In another type of test simulations, referred to as 'mirror-sym', the boundary is allowed to migrate, however a constant slope across the left and right boundary of the system (or a mirror symmetry with respect to the $\mathrm{x}$-axis) is forced, by requiring that $\eta_{\mathrm{x}=0}-\eta_{\mathrm{x}=-\Delta x}=$ $\eta_{\mathrm{x}=\Delta x}-\eta_{\mathrm{x}=0}$ and $\eta_{\mathrm{x}=\lambda_{\text {def }} / 2+\Delta x}-\eta_{\mathrm{x}=\lambda_{\text {def }} / 2}=\eta_{\mathrm{x}=\lambda_{\text {def }} / 2}-\eta_{\mathrm{x}=\lambda_{\text {def }} / 2-\Delta x}$. The results of these simulations are discussed and compared to those obtained in the presence of a retrusion later in section IV.

\section{SIMULATION RESULTS AND ANALYSIS}

The migration of an initially planar grain boundary parallel to the x-axis into the varying deformation fields was simulated. For all stored deformation energy fields shown in Fig. 3, protrusions/retrusions develop as expected along the recrystallization boundary due to the spatial variation in driving force for recrystallization. Furthermore in all cases, a steady-state grain boundary movement is reached after a certain time. The evolution of the steady state interface shape can be understood as follows. Initially the grain boundary segments where the stored deformation energy is higher advance faster forming protrusions and leaving retrusions behind in the regions where the stored deformation energy is lower. However, as long as this process continues, the local curvature at each retrusion continues to increase resulting in an extra driving 
force (locally) in the direction of grain boundary migration until a steady-state is reached where the grain boundary has obtained a shape for which the total driving force for grain boundary migration is constant along the grain boundary.

For each field, the simulated steady-state shapes of the grain boundary are shown for different values of the amplitude of the variations in Fig. 5. In all cases, for increasing values of $A_{\text {def }}$, the steady-state shape of the protrusion/retrusion becomes more asymmetric with a larger absolute curvature at the retrusions than at the protrusions as is also seen experimentally. The shape of the grain boundary in the protrusion region approaches that of a circular segment with a curvature determined by the width of the protrusion region. In contrast, the shape of the grain boundary in the retrusion region is quite different for the different variations of the stored deformation energy in the retrusion region. The shape of the grain boundary in the protrusion region is compared for 's' and 'a1', and for 's' and 'a2', in Figs. 5b and 5c, respectively.

The absolute curvatures obtained at the protrusions and retrusions and the migration rate of the grain boundary at steady-state are plotted as a function of the amplitude of the driving force $A_{\text {def }}$ in Fig. 6 considering stored deformation energies with various characteristics. For all considered variations, the curvature at the protrusions reaches asymptotically towards a constant value. Moreover, although the retrusion curvature and the migration velocity continuously increase with increasing amplitude, a kink is found in both curves near the amplitude at which the protrusion curvature saturates. The constant term $C_{d e f}$ seems not to affect the protrusion and retrusion curvatures, but results in a constant contribution to the grain boundary velocity.

A closer look at the data presented in Fig. 6a shows that, for low values of the amplitude, the protrusion curvature increases linearly with $A_{d e f}$ having a value very close to $A_{d e f} / \sigma_{g b}$ for 's' and 'a2' and close to $A_{d e f} / \sigma_{g b}(1+2 /(3 \pi))$ for 'a1'. For larger amplitudes, the protrusion curvature however stagnates at a constant value which is slightly larger than $2 / \mathrm{w}_{\text {pro }}$, with $\mathrm{w}_{\text {pro }}$ defined as shown in Figs. 5 , namely $\mathrm{w}_{\text {pro }}=\lambda_{\text {def }} / 2$ for the sinusoidal variation 's' and $\mathrm{w}_{\text {pro }}=\lambda_{\text {def }} / 3$ for the asymmetrical variations 'a1' and 'a2'. This stagnation can be related to the near circular shape of the grain boundary in the protrusion region (see Figs. 5 ), since $2 / \mathrm{w}_{\text {pro }}$ is the highest curvature a circular segment covering the full protrusion region can have. Comparison of Figs. $6 \mathrm{a}$ and $6 \mathrm{~b}$ shows that for values of $A_{\text {def }}$ below the kink $\left(A_{d e f}<A_{d e f, k i n k}\right)$, the steady-state protrusions/retrusions are almost symmetrical 
with $\rho_{r e} \approx \rho_{\text {protr }}$ and the grain boundary migration velocity $v_{g b} \approx M F_{d e f, a v}$, whereas for higher driving forces the steady-state protrusions/retrusions become more asymmetric with $\rho_{r e} \gg \rho_{p r o}$ and $v_{g b} \gg M F_{d e f, a v}$, with $F_{d e f, a v}$ the average stored deformation energy.

The relation $A_{d e f, k i n k}=2 \sigma_{g b} / \mathrm{w}_{\text {pro }}$, which defines the amplitude at which the steady-state protrusion curvature saturates for a given length scale of the variations in the deformation microstructure, is plotted in Fig. 7 assuming a grain boundary energy corresponding to that of Al. For all amplitudes of the variations in stored deformation energy $A_{d e f}$ above this curve a highly asymmetrical grain boundary shape with $\rho_{r e} \gg \rho_{p r o}$ will develop, whereas for values of $A_{d e f}$ below the curve, the grain boundary shape will only develop small nearly symmetric fluctuations $\left(\rho_{\text {re }} \approx \rho_{\text {protr }}\right)$. This curve suggests that there is a lower limit on the length scale of the variations in stored deformation energy below which typical asymmetrical protrusions/retrusions cannot form. For example, to allow formation of protrusions and retrusions a sinusoidal variation with wavelength $200 \mathrm{~nm}\left(\mathrm{w}_{\text {pro }}=100 \mathrm{~nm}\right)$ would require a stored deformation energy density of approximately $6.4 \mathrm{MJ} / \mathrm{m}^{3}$ at the protrusion, which is unrealistically high considering the stored deformation energies measured for $\mathrm{Al}^{5}$, which are of the order of $0.6 \mathrm{MJ} / \mathrm{m}^{3}$. For variations with a wavelength of $1 \mu \mathrm{m}$ and larger, such as those shown in Fig. 2, however, protrusions/retrusions are predicted to develop.

Given this stagnation of the protrusion curvature for large amplitudes, the kink in the curves of the retrusion curvature and migration velocity can be understood as follows. At steady-state the velocity along the $y$-direction is constant along the grain boundary, therefore the protrusion and retrusion curvatures are related as

$$
v_{\text {protr }}=M\left(2 A_{\text {def }}-\sigma_{g b} /\left|\rho_{\text {protr }}\right|+C_{d e f}\right)=v_{r e}=M\left(\sigma_{g b} /\left|\rho_{r e}\right|+C_{d e f}\right)
$$

for the energy fields 's' and 'a1', and as

$$
\begin{gathered}
v_{\text {protr }}=M\left(2 A_{d e f}-\sigma_{g b} /\left|\rho_{\text {protr }}\right|+C_{d e f}\right) \\
=v_{r e}=M\left(0.5 A_{d e f}+\sigma_{g b} /\left|\rho_{r e}\right|+C_{d e f}\right)
\end{gathered}
$$

for 'a2', with $M$ the grain boundary mobility and where it is assumed that the driving force for grain boundary movement consists of a contribution from the local stored deformation energy density and a contribution due to the local curvature. Therefore, for values of $A_{d e f}$ 
below the kink (where $1 /\left|\rho_{\text {protr }}\right| \approx A_{\text {def }} / \sigma_{g b}$ for 's' and 'a2' and $1 /\left|\rho_{\text {protr }}\right| \approx A_{\text {def }} / \sigma_{g b}(1+$ $2 /(3 \pi))$ for 'a1') the retrusion curvature should be very close to $A_{d e f} / \sigma_{g b}$ for the field 's', to $A_{d e f} /\left(2 \sigma_{g b}\right)$ for 'a2' and to $A_{d e f} / \sigma_{g b}(1-2 /(3 \pi))$ for 'a1', and for values of $A_{d e f}$ above the kink ( where $1 /\left|\rho_{\text {protr }}\right| \approx 2 / \mathrm{w}_{\text {pro }}$ for all 3 fields) the retrusion curvature should be very close to $2 A_{d e f} / \sigma_{g b}-2 / \mathrm{w}_{\text {pro }}$ for the fields 's' and 'a1' and to $1.5 A_{d e f} / \sigma_{g b}-2 / \mathrm{w}_{\text {pro }}$ for 'a2', as is the case for the data shown in $6 \mathrm{~b}$. Moreover, for all three forms of the deformation field, the steady-state grain boundary velocity $v_{g b}$ (Fig. 6c) is very close to $M F_{a v}\left(=M\left(A_{d e f}+C_{d e f}\right)\right.$ for 's' and 'a2' and $=M\left(A_{d e f}(1-2 /(3 \pi))+C_{d e f}\right)$ for 'a1') for $A_{d e f} \lesssim A_{d e f, k i n k}$, and to $M\left(2 A_{\text {def }}-2 \sigma_{\text {gb }} / \mathrm{w}_{\text {pro }}\right)$ for larger amplitudes, in agreement with equations (13) and (14).

It also follows from relations (13) and (14) that the 3 relations plotted in Fig. 6 scale with $\mathrm{w}_{\text {pro }}$ and $1 / \sigma_{g b}$ for a fixed form of heterogeneous deformation energy field and value of $C_{d e f}$. An example is shown in Fig. 8 for the sinusoidal variation 's'. For all three deformation fields, the scaled protrusion curvature $1 /\left|\rho_{\text {pro }}^{*}\right|$ stagnates at $A_{\text {def }}^{*} \approx 2.2$, which is slightly larger than the scaled curvature of a half circle covering the protrusion region.

The simulations suggest that the steady-state migration velocity of the boundary is mostly determined by characteristics of the heterogeneous deformation energy field in the protrusion region only, especially at large amplitudes of the stored deformation energy field. Whereas, in the retrusion region, the boundary merely adapts its local curvature to obtain the local driving force required to obtain the migration velocity dictated by the variations in the protrusion region. To study the effect of the presence of a retrusion on the shape and migration behavior of the protrusion, the steady-state curvatures along the boundary in the protrusion region are plotted in Fig. 9 for the 3 considered variations of stored deformation energy field together with those obtained for the configuration with a migrating protrusion only ('mirror-sym'), for equal $\sigma_{g b}, A_{d e f}$ and $\mathrm{w}_{\text {pro }}$ in the simulations. The comparison shows that, for large amplitudes (Fig. 9a), the curvatures (and hence the shape) of the grain boundary at and near the protrusion tip are indeed hardly affected by the presence/absence of a retrusion. Only near the boundaries of the protrusion region the grain boundary shape is slightly affected by the characteristics of the retrusion region. Since for a given $A_{d e f}$ and $\sigma_{g b}$, the grain boundary velocity is directly related to the curvature at the protrusion tip through $v=M\left(A_{d e f}-\sigma_{g b} /\left|\rho_{\text {pro }}\right|\right)$, an equal grain boundary migration velocity was obtained for the 4 configurations. In the low amplitude regime (Fig. 9b), however, the curvature at the tip of the protrusion $1 /\left|\rho_{\text {pro }}\right|$ seems to be determined by the average stored deformation 
energy, such that the grain boundary migration velocity equals $v_{g b}=M F_{d e f, a v}$.

Although the precise shape of the boundary at steady state depends on the details of the local variations in stored deformation energy, any infinite 1-dimensional variation (in addition to those used in this study) will, after a certain transition time, lead to steady-state migration, at which the velocity is determined by the average stored deformation energy for $A_{d e f}$ below a certain value and by the characteristics of the stored deformation energy field in front of the protrusion for $A_{d e f}$ above a certain value. Only one exception exists to this behaviour, namely the case of a discontinuous variation with zero-energy in the low-energy regions and a constant stored deformation energy $A_{d e f}$ in the high energy region. In this case, the protrusion/retrusion will continuously elongate. However, this is a very artificial situation, as some defects will always be present in the low energy matrix regions between the dislocation boundaries. As soon as even a very small amount of energy is present in the low energy region, migration of the elongated grain boundary parts, namely those parallel (or almost parallel) with the variations, towards the tip of the retrusion (i.e. perpendicular to the overall migration rate) is then possible due to a difference in stored deformation energy between the defect-free recrystallized region and the nonrecrystallized region with low stored deformation energy, increasing the local curvatures at the retrusion. By this mechanism, finally steady-state migration along the y-direction can be reached, although it may require a long migration distance in the case of a wide region with very low energy density.

An important insight from the model is that for large amplitudes of the driving force the migration velocity depends on the distribution of the stored energy in the deformation field. For $A_{d e f} \lesssim A_{d e f, k i n k}$, the grain boundary migration velocity is the same as that when the same amount of stored energy is distributed uniformly, according to $v_{g b}=M F_{d e f, a v}$. However, for $A_{d e f} \gtrsim A_{d e f, k i n k}$ the migration velocity is systematically higher than for the case where the same amount of deformation energy is homogeneously distributed in the deformed material. The magnitude of the increase in velocity depends on the form of the energy distribution.

Experimentally, a direct comparison of the migration velocity into deformed structures with equal average values but different spatial distributions of the stored energy is not 
possible. However, for steady state migration the amount of deformation energy released when the grain boundary advances a unit amount of distance is the same in the case of either a heterogeneous or homogeneous distribution of the deformation energy (namely $A_{\text {def }}$ times the grain boundary length). This implies therefore that the higher velocity can be related to a net curvature force in the direction of the boundary migration arising from the protrusions and retrusions.

From an experimental perspective the net curvature (i.e. the local curvature projected on the direction of grain boundary movement in every point integrated along the grain boundary: $\int(1 / \rho) \mathbf{n} \cdot \mathbf{e}_{\mathbf{y}} \mathrm{dx}$ with $\mathbf{n}$ the local unit vector perpendicular to the grain boundary, $e_{y}$ the unit vector in the direction of grain boundary motion and $\mathrm{x}$ the coordinate along the direction perpendicular to grain boundary motion ) can therefore be used as a measure of the effect of the protrusions and retrusions on the migration velocity of a recrystallization interface. As a first validation of the proposed simulation approach, we verified that the magnitude of the net curvature seen in the current model is of the same magnitude as that seen experimentally. For example in a sample of $50 \%$ deformed $\mathrm{Al}$ annealed at $250{ }^{\circ} \mathrm{C}$ for 10 minutes, a net curvature in the direction of migration of the order $0.5-0.8 \mu \mathrm{m}^{-1}$ was found. In the phase-field simulations with a stored deformation energy ${ }^{28}$ of $0.6 \mathrm{MJ} / \mathrm{m}^{3}$ and grain boundary energy of $\sigma_{g b}=0.32$ $\mathrm{J} / \mathrm{m}^{2}$ (for $\mathrm{Al}$ ), and a 1-dimensional sinusoidal variation in the deformation energy (of form 's') with wavelength $\lambda_{d e f}=6.4 \mu \mathrm{m}$, a net curvature value of $0.67 \mu \mathrm{m}^{-1}$ is obtained. In real deformation microstructures, the steady-state shape may not always be reached due to the finite length of the dislocation boundaries. This comparison however shows that in the considered material most of the protrusions/retrusions could develop curvatures (and a shape) close to the steady-state curvatures and shape obtained in the simulations.

Qualitatively, the increase in grain boundary velocity could also be predicted directly using the principle of fastest energy dissipation. The spatial variations in deformation energy density namely introduce extra degrees of freedom which allow the grain boundary to adapt its shape and move faster to achieve faster energy dissipation. The actual magnitudes of the increase in grain boundary velocity are however correlated in a complicated way with details of the spatial variations in the stored deformation energy and grain boundary properties. It is therefore required to use a model that considers the migration of recrystallization 
boundaries locally to quantify the effect.

\section{CONCLUSIONS}

In summary we have proposed a phase-field approach for boundary migration during recrystallization in the presence of a varying deformation field. The model has been used to characterize quantitatively protrusion/retrusion formation during boundary migration into a deformation field with 1-dimensional sinusoidal variations of both symmetric and asymmetric form assumed to represent a single set of dislocation boundaries. The model is able to reproduce the experimentally observed asymmetry between the protrusion and retrusion curvatures. Additionally, the simulations show that the protrusion curvature as a function of the amplitude of the variations in the deformation energy field saturates for amplitudes larger than $A_{d e f, k i n k}$ at which the protrusion curvature is approximately $2 / \mathrm{w}_{\text {pro }}$ and at which value there is also a kink in the curves of the retrusion curvature and migration velocity.

Moreover, for larger amplitudes $\left(A_{d e f} \gtrsim A_{d e f, k i n k}\right)$ of the variations in the deformation energy field, the boundary moves at a considerably higher velocity, determined by characteristics in front of the protrusion only, than when the same driving force is distributed homogeneously in the deformed material, and this can be related to a net driving force due to curvature along the boundary. The net driving forces due to curvature obtained in the simulations are similar in magnitude to those calculated from experimental micrographs.

The modeling technique presented here can be applied using alternative more complicated forms of spatial variations in the stored deformation energy in 2 and 3 dimensions, including localized discontinuous descriptions, and extended to account for e.g. orientation or composition dependent grain boundary properties ${ }^{21}$. This type of modelling is essential for interpretation of experimental observations of local boundary migration during recrystallization and for suggesting new experiments.

\section{ACKNOWLEDGMENTS}

NM acknowledges the Research Foundation - Flanders for grant No. V.4.280.10N - VC - 8217. AG, YBZ and DJJ acknowledge support from the Danish National Research Foundation and the National Natural Science Foundation of China (Grant No. 50911130230) 


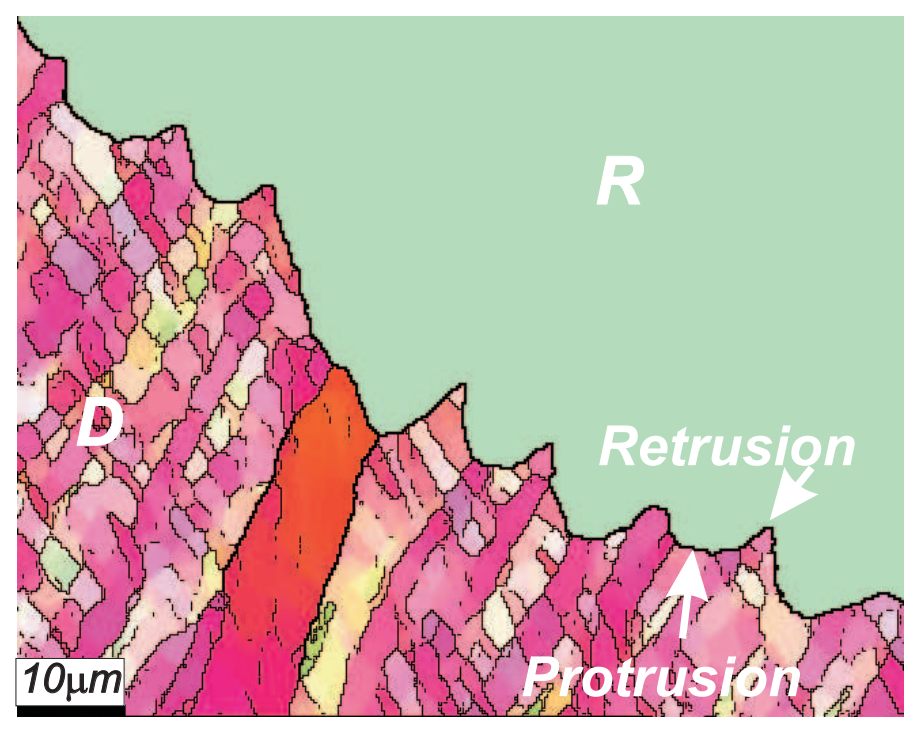

FIG. 1. Micrograph showing the presence of protrusions and retrusion on a migrating recrystallization boundary as obtained in pure aluminium (99.996\%), cold rolled to $50 \%$ in thickness at room temperature, followed by annealing at $250{ }^{\circ} \mathrm{C}$ for 10 minutes. The letters $\mathrm{R}$ and $\mathrm{D}$ refer to recrystallized and deformed, respectively. The colors refer to the $\mathrm{RD}$ inverse pole figure.

for the Danish-Chinese Center for Nanometals. NM and AG also acknowledge the funding for Bilateral Scientific Cooperation between Tsinghua University \& KU Leuven (Grant BIL10/19T). We also thank Liz Holm for the useful idea of studying the shape of a single protrusion.

* nele.moelans@mtm.kuleuven.be

† awgodfrey@mail.tsinghua.edu.cn

$\ddagger$ yubz@dtu.dk

$\S$ doje@dtu.dk

1 N. Hansen and D. Juul Jensen, Materials Science and Technology 27, 1229 (2011).

2 X. Huang and W. G, Philos. Mag. 87, 5189 (2007).

3 A. Godfrey, D. Juul Jensen, and N. Hansen, Acta Mater. 46, 823 (1998).

4 A. Godfrey, D. Juul Jensen, and N. Hansen, Acta Mater. 49, 2429 (2001).

5 A. Godfrey, N. Hansen, and D. Juul Jensen, Metall. Mater. Trans. A 38A, 2329 (2007). 


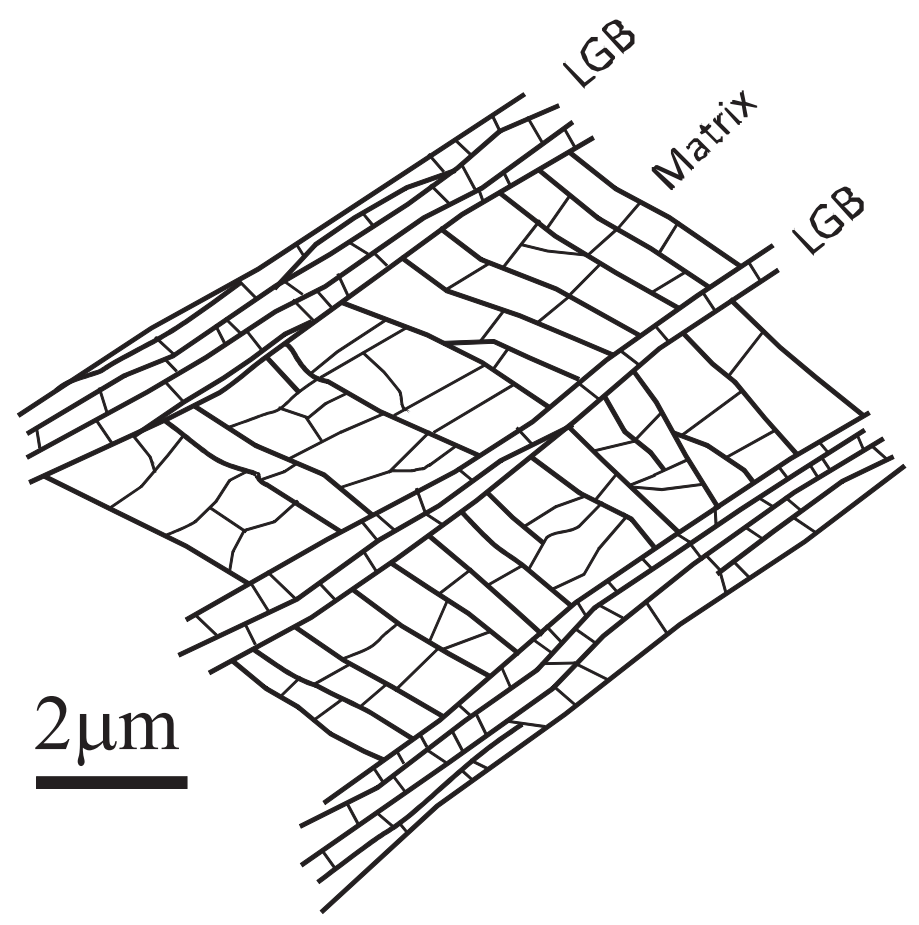

FIG. 2. Sketch (taken from ${ }^{4,5}$ ) illustrating the arrangement of the dislocation boundaries for a single crystal (Al) with $\mathrm{C}$ orientation deformed to $78 \%$ reduction, showing local variations in the microstructure on the scale of a few $\mu \mathrm{m}$, i.e. the microstructure consists of localized glide bands (LBG's) of high stored deformation energy density with matrix regions with lower stored deformation energy density in between.

6 P. Beck and P. Sperry, J. Appl. Phys. 21, 150 (1950).

7 S. Schmidt, S. F. Nielsen, C. Gundlach, L. Margulies, X. Huang, and D. Juul Jensen, Science 305, 229 (2004).

8 W. Xu, M. Z. Quadir, and M. Ferry, Metall. Mater. Trans. A 40A, 1547 (2009).

9 Y. B. Zhang, A. Godfrey, Q. Liu, W. Liu, and D. Juul Jensen, Acta Mater. 57, 2631 (2009).

10 M. A. Martorano, H. R. Z. Sandim, M. A. Fortes, and A. F. Padilha, Scripta Mater. 56, 903 (2007).

11 Y. B. Zhang, A. Godfrey, and D. Juul Jensen, CMC-Computers Materials \& Continua 14, 197 (2009).

12 Y. B. Zhang, A. Godfrey, and D. Juul Jensen, Scripta Mater. 64, 331 (2011).

13 M. A. Martorano, M. A. Fortes, and A. F. Padilha, Acta Mater. 54, 2769 (2006).

14 R. B. Godiksen, Z. T. Trautt, M. Upmanyu, J. Schiotz, D. Juul Jensen, and S. Schmidt, Acta 

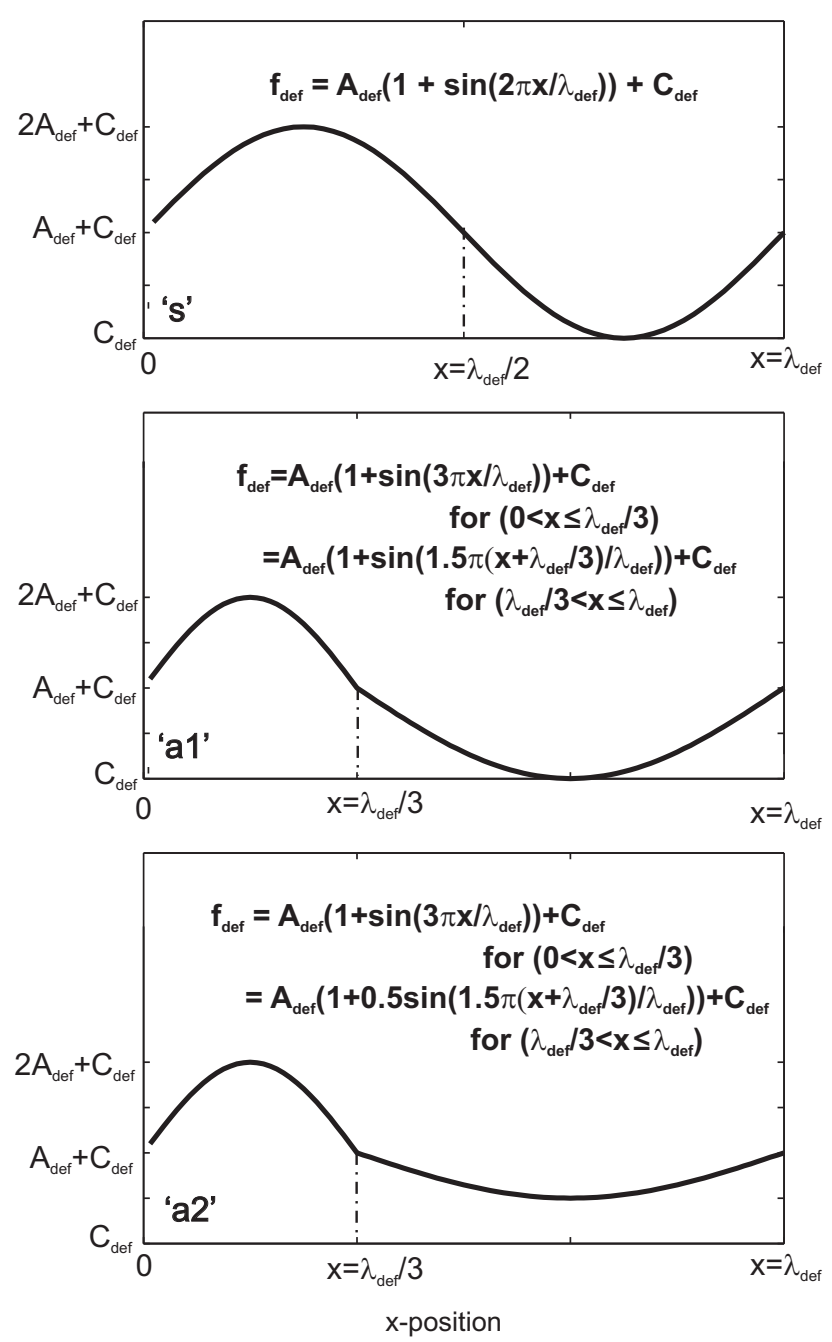

FIG. 3. The 3 forms of varying deformation energy fields $f_{\text {def }}$ considered in this work, referred to as 's', 'a1' and 'a2'. The average stored deformation energy $F_{d e f, a v}$ equals $A_{d e f}+C_{d e f}$ for 's' and 'a2' and $(1-2 /(3 \pi)) A_{d e f}+C_{d e f}$ for 'a1'.

Mater. 55, 6383 (2007).

15 R. B. N. Godiksen, S. Schmidt, and D. Juul Jensen, Modelling. Simul. Mater. Sci. Eng. 16, 065002:1 (2008).

16 S. Sreekala and M. Haataja, Phys. Rev. B 76, 094109:1 (2007).

17 N. Moelans, B. Blanpain, and P. Wollants, CALPHAD - Comp. Coupl. Phase Diagrams and Thermochemistry 32, 268 (2008).

18 L.-Q. Chen and W. Yang, Phys. Rev. B 50, 15752 (1994).

19 N. Moelans, B. Blanpain, and P. Wollants, Phys Rev Lett 101, 025502 (2008). 


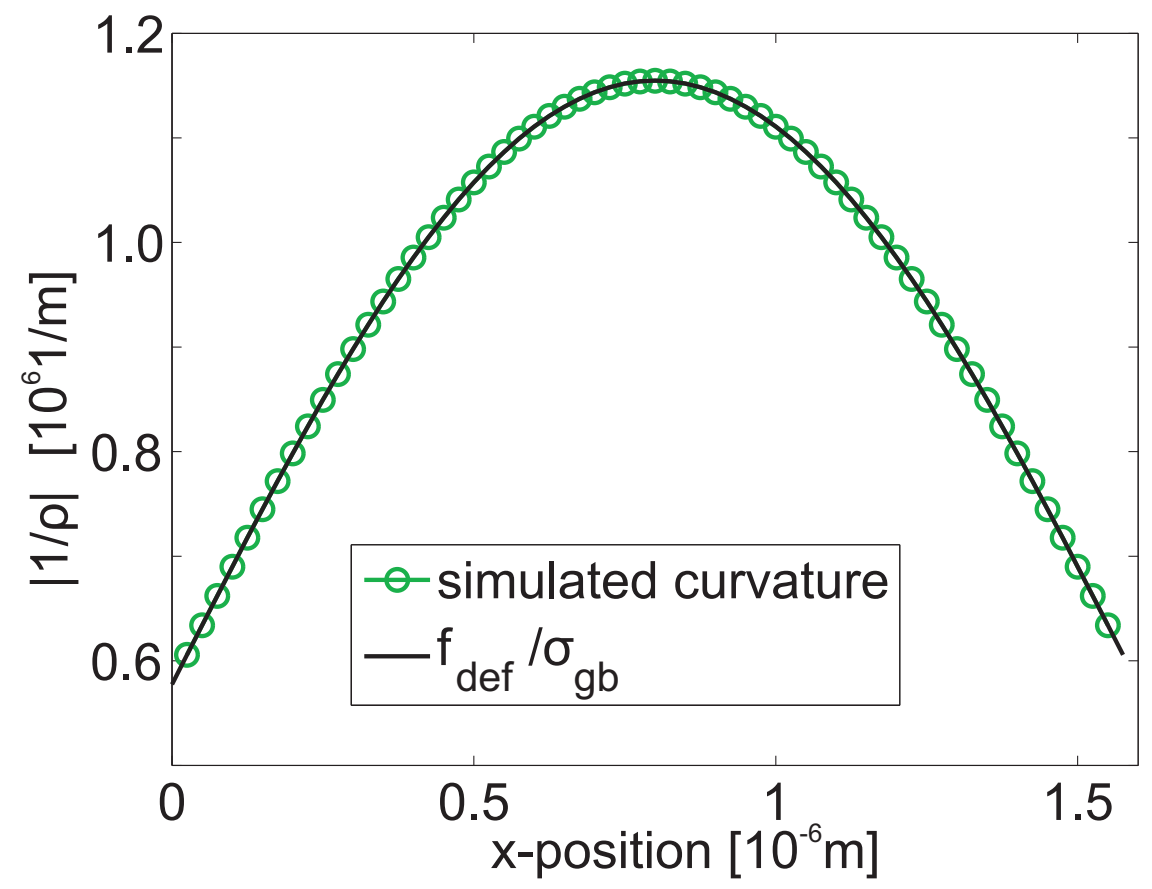

FIG. 4. Simulated curvatures along the grain boundary compared with the values expected from a force balance in the y-direction for configuration 'static' (as described in section III) with $A_{d e f}$ $=0.5 \mathrm{MJ}, \sigma_{g b}=0.87 \mathrm{~J} / \mathrm{m}^{2}$ and $\lambda_{\text {def }}=3.2 \mu \mathrm{m}$.

20 N. Moelans, B. Blanpain, and P. Wollants, Phys. Rev. B 78, 024113 (2008).

21 N. Moelans, Acta Mater. 59, 1077 (2011).

22 A. Godfrey, D. Juul Jensen, and N. Hansen, Acta Mater. 46, 835 (1998).

23 W. T. Read and W. Shockley, Phys. Rev. B 78, 275 (1950).

24 T. Knudsen, W. Q. Cao, A. Godfrey, Q. Liu, and N. Hansen, Metall. Mater. Trans. A 39A, $430(2008)$.

25 N. Moelans, F. Wendler, and B. Nestler, Comp. Mater. Sci. 46, 479 (2009).

26 L. N. Trefethen, Spectral methods in MATLAB (SIAM, Philadelphia, PA, 2000).

27 N. Moelans, Ph.D. thesis, K.U.Leuven (2006).

28 Q. Liu, D. Juul Jensen, and N. Hansen, Acta Mater. 46, 5819 (1998). 




(a)

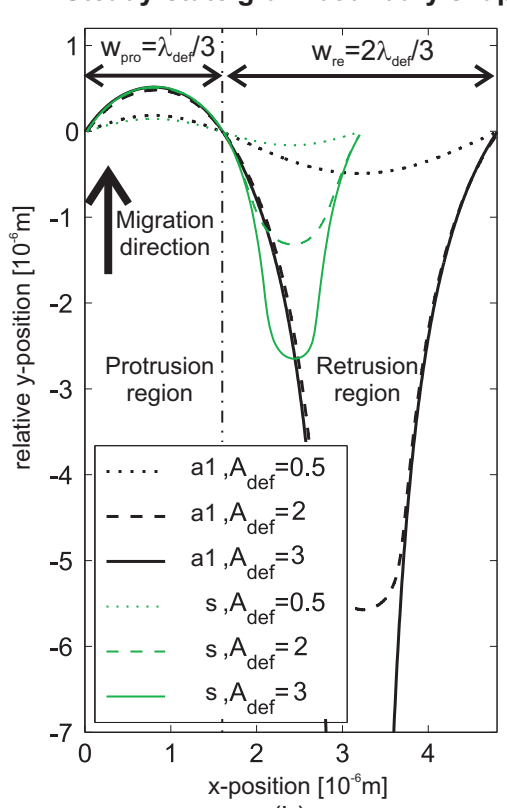

(b)

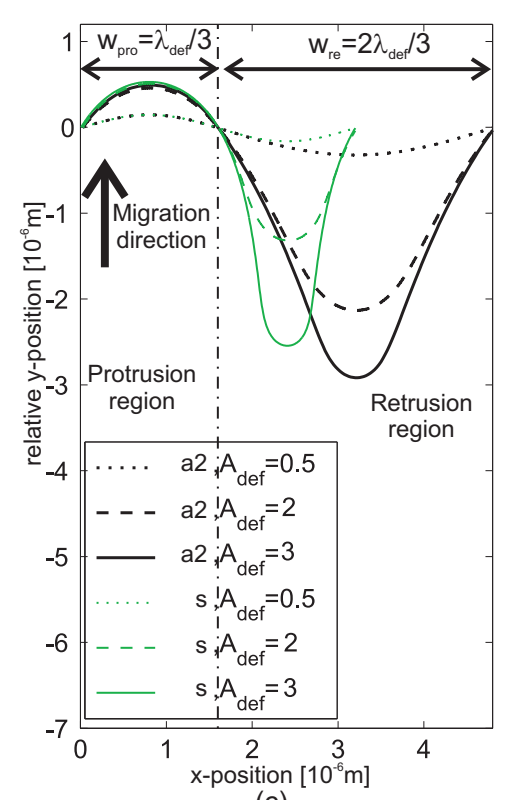

(c)

FIG. 5. (a) Steady-state shapes of protrusion/retrusions obtained for varying deformation energy fields of the form 's' with $\lambda_{d e f}=3.2 \mu \mathrm{m}$ and for two values of the grain boundary energy $\sigma_{g b}$ and different values of the amplitude $A_{\text {def }}$ (expressed in $\mathrm{MJ} / \mathrm{m}^{3}$ ). (b) and (c) Steady-state shapes of protrusion/retrusions obtained for varying deformation energy fields of the form 'a1' (b) and 'a2' (c) with $\lambda_{\text {def }}=4.8 \mu \mathrm{m}$ and 's' with $\lambda_{\text {def }}=3.2 \mu \mathrm{m}$ for a grain boundary energy $\sigma_{g b}=0.87 \mathrm{~J} / \mathrm{m}^{2}$ and different values of the amplitude $A_{d e f}$. For explanation of 's', 'a1' and 'a2' see Fig. 3 

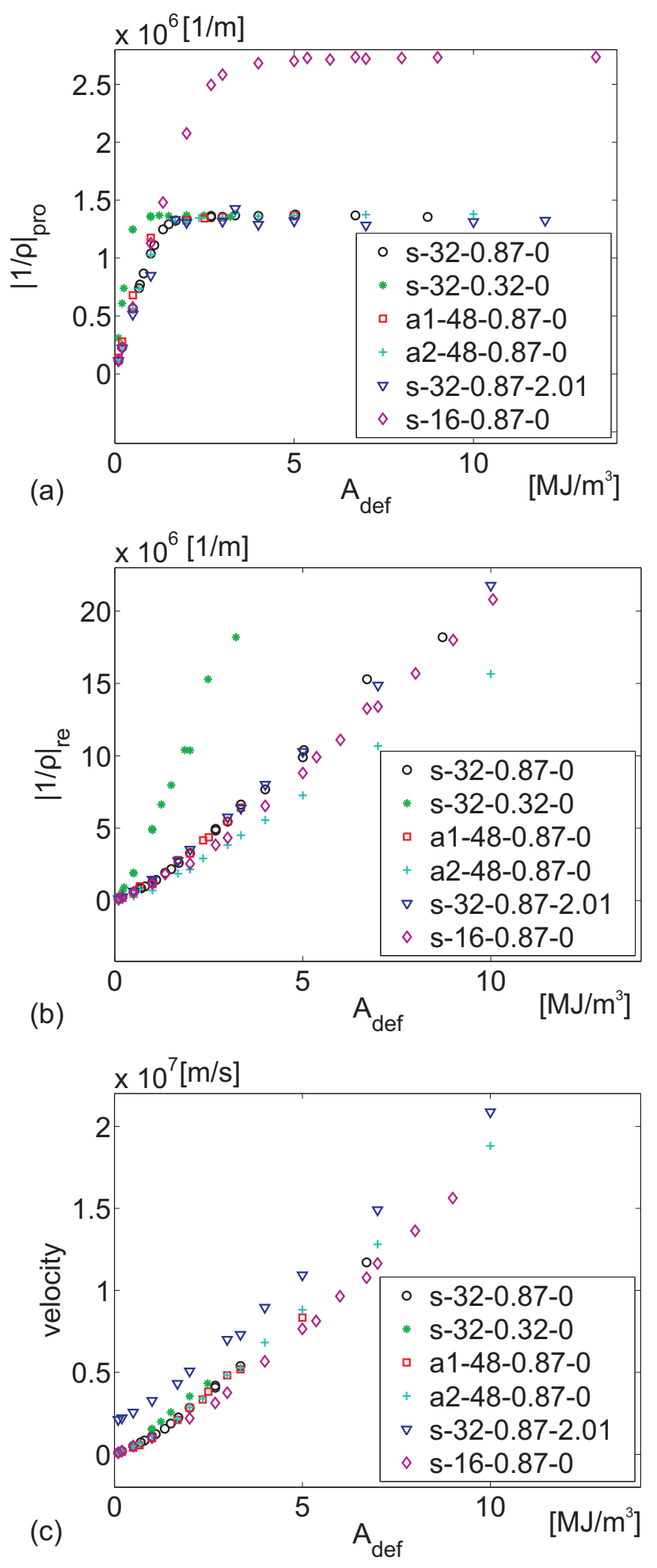

FIG. 6. (a) Absolute local curvatures at the protrusion $\left|1 / \rho_{\text {pro }}\right|$ and (b) retrusion $\left|1 / \rho_{\text {re }}\right|$ and (c) migration velocity $v_{g b}$, as a function of the amplitude of the varying deformation energy fields $A_{d e f}$ for the 3 forms shown in Fig. 3 and different wavelengths $\lambda_{d e f}$, grain boundary energies $\sigma_{g b}$ and offset values $C_{d e f}$. The entries in the legend are 'form of variation $-\lambda_{d e f}-\sigma_{g b}-C_{d e f}$ '. 


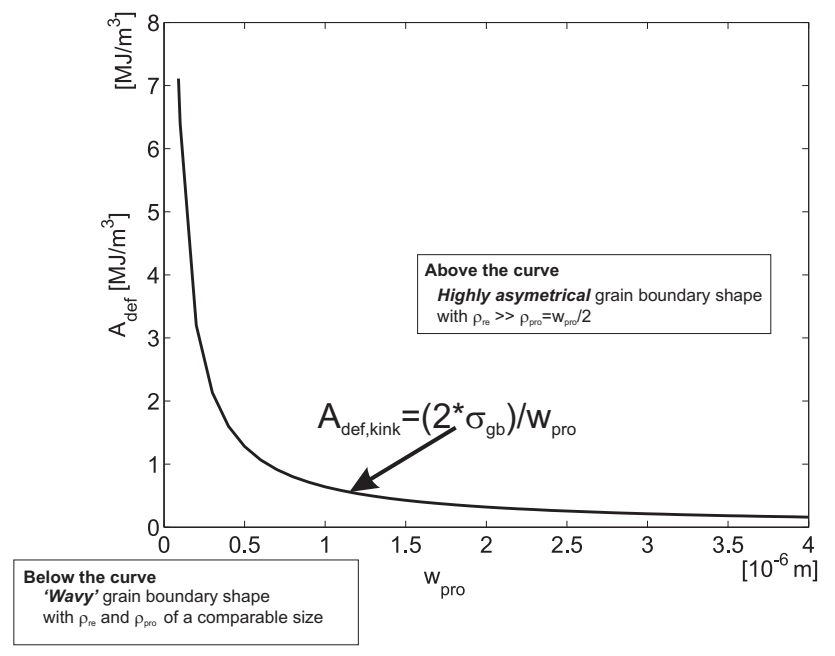

FIG. 7. Amplitude of the variations in stored deformation energy density at which the protrusion curvature saturates, $A_{d e f, k i n k}$, as a function of $\mathbf{w}_{\text {pro }}$ ( defined in Figs 5) for the (symmetrical or asymmetrical) sinusoidal fields considered in this study and assuming a grain boundary energy equal to $0.32 \mathrm{~J} / \mathbf{m}^{2}$. For a given $\mathbf{w}_{\text {pro }}$, an highly asymmetrical grain boundary shape develops for all $A_{d e f}$ larger than $A_{\text {def,kink}}$. 

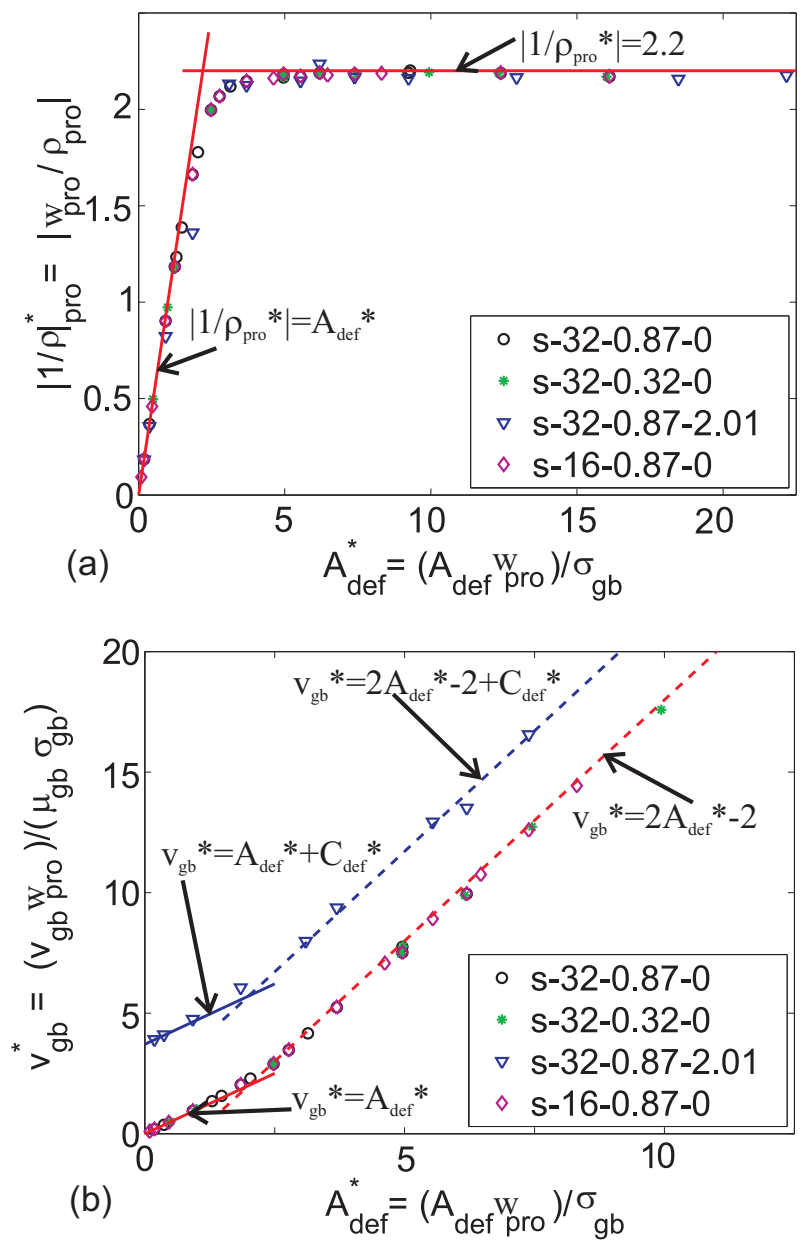

FIG. 8. (a) Scaled absolute protrusion curvature $\left|1 / \rho_{\text {pro }}^{*}\right|$ and (b) scaled velocity $v_{g b}^{*}$ as a function of the scaled amplitude $A_{d e f}^{*}$ obtained for varying deformation energy fields of the form 's' and different wavelengths $\lambda_{d e f}$, grain boundary energies $\sigma_{g b}$ and offset values $C_{d e f}$, with $C_{d e f}^{*}=\left(C_{d e f} \mathrm{w}_{p r o}\right) / \sigma_{g b}$. The entries in the legend are 'form of variation - $\lambda_{d e f}-\sigma_{g b}-C_{d e f}$ '. 

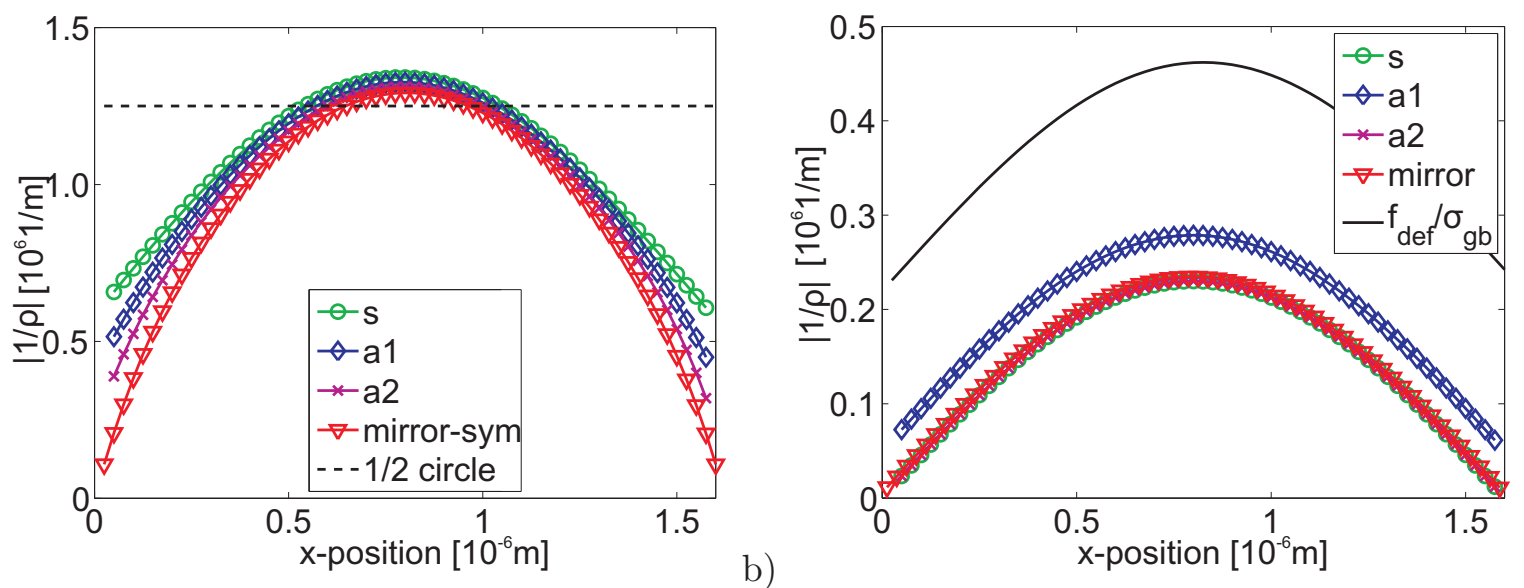

FIG. 9. Simulated steady-state grain boundary curvature in the protrusion region for the 3 variations in stored deformation energy 's', 'a1' and 'a2' (for explanation of 's', 'a1' and 'a2' see Fig. 3) and for the configuration 'mirror-sym' with a migrating protrusion only ( see section III for a detailed description) for equal conditions in the protrusion region, but with different characteristics for the retrusion region. a) For $\mathrm{w}_{\text {pro }}=1.6 \mu \mathrm{m}, \sigma_{g b}=0.87 \mathrm{~J} / \mathrm{m}^{2}$ and $A_{\text {def }}=2 \mathrm{MJ} / \mathrm{m}^{3}$, for which $A_{d e f}>A_{d e f, k i n k}$. The constant curvature of half a circle covering the protrusion region is added in broken line. b) For $\mathrm{w}_{\text {pro }}=1.6 \mu \mathrm{m}, \sigma_{g b}=0.87 \mathrm{~J} / \mathrm{m}^{2}$ and $A_{d e f}=0.2 \mathrm{MJ} / \mathrm{m}^{3}$, for which $A_{d e f}<A_{d e f, k i n k}$. The full line gives the curvature expected for a 'static' grain boundary, showing that the grain boundary curvature is affected by the kinetics. 